\title{
La teoría de los diferenciales de Salomon Maimon, la pregunta quid juris y la posibilidad de la metafísica como ciencia
}

\author{
Salomon Maimon's Theory of Differentials, \\ the quid juris Question and the Possibility \\ of Metaphysics as a Science
}

\author{
Hernán PRINGE \\ CONICET-UBA (Argentina) / Universidad Diego Portales, Santiago (Chile)
}

Recibido: 02-03-2015

Aceptado: 24-09-2015

\section{Resumen}

Este trabajo estudia la teoría de los diferenciales de Salomon Maimon como respuesta a la cuestión quid juris y a la vez como clave para la fundamentación de la posibilidad de la metafísica como ciencia. Se reconstruye primero la crítica de Maimon al tratamiento kantiano de la pregunta quid juris. Luego, se analiza la respuesta del propio Maimon a esa pregunta, para establecer finalmente cómo tal respuesta abre el camino para la explicación de la posibilidad de la metafísica como ciencia.

Palabras clave: Diferencial, idealismo trascendental, metafísica, pregunta, quid juris.

\section{Abstract}

This paper studies Salomon Maimon's theory of differentials both as an answer to the quid juris question and as a key to the foundation of the possibility of metaphysics as a science. Firstly, we reconstruct the Maimonian criticism to the Kantian approach to the quid juris question. Later, we analyze Maimon's own response to 
that question in order to finally establish how such answer paves the road to the explanation of the possibility of metaphysics as a science.

Keywords: Differential, metaphysics, quid juris question, transcendental idealism.

\section{Introducción}

En su Ensayo acerca de la filosofía transcendental1, Salomon Maimon propone una clave para la justificación de la aplicabilidad de conceptos puros a objetos empíricos, o, en otras palabras, Maimon presenta su propia respuesta a la pregunta kantiana quid juris ${ }^{2}$. Maimon sostiene que la filosofía de Kant no es capaz de dar una solución satisfactoria a tal problema y que ésta sólo podría alcanzarse desde una posición leibnizo-wolffiana. ${ }^{3}$ Más concretamente, la heterogeneidad que Kant plantea entre entendimiento y sensibilidad debería ser abandonada en favor de una homogeneidad basada en el origen común de concepto y dato sensible. Según Maimon, el contenido sensible se relacionaría con el concepto tal como lo hacen lo generado y la ley de su generación. Maimon introduce aquí la teoría de los diferenciales para fundamentar la explicación de tal aplicabilidad empírica de conceptos a priori. La relación entre una cierta función y su función derivada resultaría análoga a la relación que existe entre lo sensible y la ley intelectual de su producción. Puesto que esta ley es determinada, lo sensible perdería su carácter de pasivamente recibido y, por lo tanto, de heterogéneo respecto de los conceptos del entendimiento. Así, el problema que la deducción trascendental y el esquematismo kantianos deben resolver desaparecería y se alcanzaría finalmente la buscada solución de la cuestión quid juris.

Pero la teoría de los diferenciales permitiría no sólo explicar la aplicabilidad de conceptos puros a objetos empíricos, sino, ante todo, responder a la pregunta por la posibilidad de la metafísica como ciencia. La posibilidad de la metafísica se basaría en una interpretación filosófica de la relación matemática entre integrales y derivadas, de modo que el teorema fundamental del cálculo resultaría la expresión matemática de la relación fundamental de la metafísica: la que existe entre lo sensible y lo suprasensible. 4

\footnotetext{
${ }^{1}$ Maimon (2004). De aquí en adelante nos referiremos a esta obra como VT. Las traducciones de las citas de Maimon son nuestras.

2 VT, 11.

3 VT, 40.

4 La importancia de la teoría de los diferenciales para el establecimiento de la posibilidad de la metafísica como ciencia no ha sido suficientemente estudiada por los comentadores. La mayoría de ellos señalan el rol de la teoría de los diferenciales en la respuesta maimoniana a la cuestión quid juris, pero no en lo que concierne a la posibilidad de la metafísica. Véase: Atlas (1964) 109 -123; Cassirer (2000)
} 
Nos proponemos en el presente trabajo discutir la teoría de los diferenciales presentada en el Ensayo desde esta doble perspectiva: como respuesta a la cuestión quid juris y como clave para la fundamentación de la posibilidad de la metafísica como ciencia. Comenzaremos reconstruyendo la crítica de Maimon al tratamiento kantiano de la pregunta quid juris (1.). Luego, analizaremos la respuesta de Maimon a esa pregunta (2.), para, finalmente, estudiar cómo tal respuesta brinda a la vez la clave para la comprensión de la posibilidad de la metafísica como ciencia (3.).

\section{La interpretación de Maimon de la deducción kantiana: la pregunta quid juris}

Maimon sostiene que, de acuerdo con la doctrina de Kant, para el conocimiento de objetos determinados son necesarios conceptos a priori, intuiciones empíricas y, además, la aplicación de los primeros sobre las segundas. ${ }^{5}$ Sin conceptos a priori sería posible intuir objetos determinados, pero de ningún modo pensarlos. En tal situación, dichos objetos serían sólo objetos del intuir pero no del entendimiento. Los objetos meramente intuidos no serían nada para el yo y por lo tanto no pertenecerían a $m i$ experiencia. Por el contrario, con conceptos a priori y sin intuiciones podríamos pensar objetos en general, pero no conoceríamos ningún objeto, pues no tendríamos concepto de objeto determinado alguno. Finalmente, si tuviéramos tanto conceptos a priori como intuiciones empíricas, pero los objetos intuidos no fueran efectivamente subsumidos bajo los conceptos del entendimiento, tendríamos las

93 - 100; Duffy (2004); Guéroult (1929) 59 - 86; Hoyos (2001) 329 - 340; Kauferstein (2006) 309 348; Kroner (1921) 353 - 356. Thielke sostiene que la teoría de los diferenciales está diseñada para brindar un modelo de conocimiento tal que lo dado sea reconducido a una función activa o espontánea del pensamiento mismo. Thielke (2003) 111. En su discusión de la teoría de los diferenciales, Bergmann, Kuntze y Zac se refieren muy brevemente a la cuestión de la posibilidad de la metafísica, sin desarrollarla: Bergmann (1967) 270; Kuntze (1912) 334; Zac (1988) 169 y 171. Según Kuntze, el problema a resolver por la teoría de los diferenciales es más bien el del fundamento de la particularidad de las cosas empíricas, una vez que Maimon rechaza la afección por parte de cosas en sí: Kuntze (1912) 331. Buzaglo subraya el interés de Maimon por restaurar la dignidad perdida de la metafísica, pero no reconoce en la teoría de los diferenciales un rol importante en esta empresa: Buzaglo (2002) 4 y $124-128$. Ehrensperger no se refiere a la teoría de los diferenciales al discutir la pregunta por la posibilidad de la metafísica: Ehrensperger (2004) XXXV-XXXIX. Más acertada nos parece la lectura de Engstler, quien atiende a la relación entre la teoría de los diferenciales, la pregunta quid juris y el conocimiento metafísico: Engstler (1990) 182 - 189. Sin embargo, para Engstler sólo una posición platónica podría explicar la captación de la ideas propia del conocimiento metafísico, lo que resultaría incompatible con la teoría de los diferenciales: Engstler (1990) 186 - 187. Por el contrario, veremos cómo Maimon da cuenta del conocimiento metafísico mediante una interpretación fílosófica de las operaciones matemáticas de diferenciación e integración que no sólo es compatible sino que presupone su teoría de los diferenciales.

5 VT, 34. 
partes constituyentes de un conocimiento "(conceptos universales que se encuentran in concreto en objetos particulares y objetos particulares a los que conceptos universales pueden aplicárseles)" 6 , pero no conocimiento como tal. De tal modo, el conocimiento exige no sólo conceptos a priori e intuiciones empíricas, sino más bien la aplicación de los primeros a las segundas. En otras palabras, la experiencia requiere la aplicación de los conceptos puros del entendimiento a objetos empíricos determinados.

Ahora bien, dado que los categorías, en tanto a priori, y los objetos empíricos, en tanto a posteriori, son totalmente heterogéneos entre sí, 7 surge de modo inmediato la pregunta quid juris: “¿Cómo resulta comprensible que conceptos a priori del entendimiento [...] puedan suministrar determinaciones de algo a posteriori?"8 Maimon presenta la respuesta de Kant a esta pregunta del siguiente modo:

Nosotros no aplicamos estos conceptos inmediatamente a la materia de la intuición, sino sólo a su forma a priori (el tiempo) y mediante ésta a la intuición misma. Si yo por lo tanto digo " $a$ es la causa de $b$," o, "si $a$ es puesto, $b$ debe necesariamente ser puesto también," entonces no se determina $a$ y $b$ según su materia o contenido, sino según determinaciones particulares de su forma (el preceder y el suceder en el tiempo): es decir, $a$ es $a$ y no $b^{9}$ no porque aquél tiene una determinación material que éste no tiene (pues esto, en tanto es algo a posteriori, no puede ser subsumido bajo una regla a priori), sino porque $[a]$ tiene una determinación formal (el preceder) que $b$ no tiene. Y es lo mismo con $b$ : él resulta un objeto determinado, distinto de $a$, no mediante una determinación material sino mediante una determinación formal (el suceder) de la forma común a ambos (el tiempo). Lo que precede se comporta entonces aquí respecto de lo que sucede como el antecedente respecto del consecuente en un juicio hipotético. Mediante este procedimiento el entendimiento es capaz no sólo de pensar objetos en general, sino de conocer objetos determinados. 10

Veamos ahora con un poco más de detalle la argumentación de Kant que Maimon resume en el pasaje citado. En la deducción trascendental de las categorías, Kant se ocupa de mostrar que las categorías no son conceptos vacíos, sino que poseen realidad objetiva. Kant demuestra que los fenómenos se encuentran necesariamente bajo las categorías, pues de otro modo no pertenecerían a $m i$ experiencia, es decir no serían nada para el yo. Para ello, se establece que el espacio y el tiempo puros deben ser unificados mediante una síntesis llevada a cabo por la imaginación, pero reglada por las categorías. De este modo, se alcanza la unidad del espa-

\footnotetext{
$6 \mathrm{VT}, 35$.

7 VT, 35.

8 VT, 28.

${ }^{9}$ Como si dijera " $a$ es la causa y no el efecto."

10 VT, 34. Véase también VT, 28 y VT, 75.
} 
cio y el tiempo que, como meras formas de la sensibilidad, no son más que multiplicidades puras. Ahora bien, en tanto el espacio y el tiempo deben ser unificados mediante una síntesis categorial, aquello que es dado en el espacio y el tiempo (la materia que es dada en las formas de espacio y tiempo) debe ser también sintetizado en una experiencia única. Esto es así pues la materia no puede sino someterse a las condiciones que la forma le impone: de otro modo, la materia no sería dada en absoluto. De esta manera, la deducción trascendental demuestra que los fenómenos deben ser subsumidos bajo las categorías precisamente porque las categorías legislan aquella síntesis a priori de la imaginación, que unifica la forma espacio-temporal de tales fenómenos.

Así, la deducción trascendental establece que los fenómenos se subsumen necesariamente bajo las categorías. Será tarea del esquematismo determinar cómo esta subsunción necesaria puede llevarse efectivamente a cabo. El problema específico del esquematismo se plantea ante la presencia de un fenómeno en la intuición. En esa situación, nos encontramos frente a la exigencia ya establecida de subsumir ese fenómeno bajo las categorías, pero, a la vez ante la dificultad basada en la absoluta heterogeneidad de sensibilidad y entendimiento. En efecto, ¿cuáles serán las notas sensibles del fenómeno que permitirán su exigida subsunción bajo conceptos puros del entendimiento? De hecho, parecería no haber nada en el fenómeno, en tanto objeto del intuir, que posibilitara su necesaria subsunción bajo las categorías del pensar, pues receptividad y espontaneidad son irreductibles entre sí. La mutua irreductibilidad de espontaneidad y receptividad hace que se vuelva problemática la subsunción de objetos sensibles bajo conceptos puros. Este problema es propio de aquella facultad que se ocupa de subsumir un caso bajo una regla: la facultad de juzgar. Por ello, debemos abandonar la analítica de los conceptos y pasar a la analítica de los principios, que enseña a aplicar los conceptos del entendimiento a los fenómenos. ${ }^{11}$ Más concretamente, en el esquematismo, el juicio debe subsumir un objeto particular dado en la sensibilidad bajo una cierta regla universal provista por el entendimiento. Por un lado, el objeto particular a subsumir está presente en la intuición, mientras que, por el otro, la regla universal que lo subsume es una determinada categoría. Así, mientras que la deducción establecía la necesidad de la subsunción de objetos espaciotemporales bajo categorías en general, el esquematismo debe mostrar cómo esta subsunción se verifica bajo cada una de las categorías, cuando un objeto particular es dado. ${ }^{12} \mathrm{El}$ esquematismo posibilita entonces la aplicación de las categorías a fenómenos, pues indica aquellas condiciones universales pero sensibles de los mismos, gracias a las cuales ellos pueden ser subsumidos bajo cada uno de los conceptos puros del entendimiento. Tales condiciones son los esquemas. Ahora bien, la exigencia de que la condición mediadora entre concepto e intuición

11 B171. Esto lo hace notar con claridad Caimi en: Caimi (2009). Véase también: Matherne (2014).

12 Véase: Caimi (2013). 
sea a la vez universal y sensible es satisfecha si tal condición es una determinación a priori de la forma sensible de todos los fenómenos en general, tanto externos como internos. Pero esta forma es el tiempo. Por lo tanto, los esquemas resultan determinaciones trascendentales del tiempo. Estas determinaciones a priori de la forma sensible de los fenómenos establecen para cada categoría qué notas del fenómeno dado lo hacen un caso de tal regla. Es precisamente esta información la que está contenida en el esquema de cada una de las categorías. Así, por ejemplo, lo permanente en el fenómeno será subsumido bajo el concepto de substancia (es decir, aquí es la nota temporal de permanencia lo que hace apto a un fenómeno para ser subsumido bajo esa precisa categoría); o lo real, a lo cual, cuando es puesto, siempre le sigue algo diferente, será subsumido bajo el concepto de causa (y aquí es la sucesión necesaria la que oficia de nota temporal que hace apto a un fenómeno para ser subsumido bajo esta última categoría). En definitiva, mediante tales determinaciones del tiempo, el esquematismo del entendimiento realiza la tarea cuya posibilidad y necesidad había sido previamente establecida por la deducción trascendental de las categorías, a saber: la de llevar lo múltiple dado en la intuición empírica bajo la unidad de la apercepción. ${ }^{13}$ Con ello, los fenómenos dados en la sensibilidad resultan finalmente objetos también pensados por el entendimiento, según las categorías.

Maimon critica esta argumentación de Kant. Como acabamos de ver, la estrategia de Kant para explicar la necesaria aplicabilidad de las categorías a fenómenos, dada la heterogeneidad entre regla intelectual y dato sensible, es considerar a los esquemas, en tanto determinaciones a priori de la intuición pura, como representaciones mediadoras entre conceptos e intuiciones empíricas. Dado que la intuición pura es forma de la intuición empírica, los enlaces determinados de la intuición pura representados por los esquemas serán válidos también respecto de la materia que se dé en el espacio y el tiempo. Como, además, tales enlaces son enlaces categoriales, las categorías tendrán en definitiva validez respecto de objetos empíricos. Pero de este modo, para Maimon, Kant no hace más que sustituir un problema por otro, dejando abierta la cuestión. Si la dificultad inicial era la de entender la aplicabilidad de las categorías a fenómenos, debido a la heterogeneidad entre conceptos e intuiciones empíricas, ahora la dificultad que se presenta es la de explicar la aplicabilidad de las categorías a la intuición pura, pues, tal como Maimon señala, también la intuición a priori es, en tanto intuición, siempre heterogénea respecto del concepto. Es por ello que el recurso kantiano al esquematismo de las categorías resulta estéril. Así, Maimon sostiene:

El señor Kant busca evitar esta dificultad asumiendo que tiempo y espacio, así como sus posibles determinaciones, son representaciones a priori en nosotros, por lo que nosotros

$13 \mathrm{~B} 185$. 
podemos justificadamente atribuirle a la sucesión determinada en el tiempo, que es $a$ priori, el concepto de necesidad, que también es a priori. Sin embargo, dado que, como ya se ha mostrado, las intuiciones, aunque sean a priori, son no obstante heterogéneas respecto de los conceptos del entendimiento, mediante esta presuposición no avanzamos demasiado. ${ }^{14}$

Pero esto no es todo. Incluso si aceptáramos, por mor del argumento, que la aplicación de las categorías a la forma a priori del fenómeno (el tiempo) no es problemática, con ello sólo podríamos explicar la aplicación de las categorías a un objeto en general, pero no a objeto determinado alguno. En efecto, respecto de la categoría de causalidad Maimon se pregunta:

¿De qué nos sirve la regla universal a priori de que tanto $b$ como $c$ deben seguir a algo según una regla (si es que esta sucesión ha de tener realidad objetiva), si sin embargo debemos aprender de la experiencia si es $b$ o es $c$ el que en relación a $a$ debe ser subsumido bajo esa regla? ${ }^{15}$

Supongamos, nos dice Maimon, que encendemos la estufa en nuestro cuarto (evento $a$ ), para luego notar que el aire en la habitación se ha entibiado (evento $b$ ) y que afuera un copo de nieve ha caído (evento $c$ ). El principio de causalidad, que sostiene que el carácter objetivo de los eventos $b$ y $c$ depende de que ellos sigan a algún otro según una regla, deja indeterminado si el evento $a$ es la causa de alguno de ellos o no. No es ningún principio a priori sino la experiencia misma la que nos muestra que al calor de la estufa siempre le sigue la tibieza del aire de la habitación, pero no siempre la caída de un copo de nieve afuera, que puede incluso suceder antes de que yo encienda la estufa. Es entonces la experiencia la que nos permite conectar causalmente los eventos $a$ y $b$. Así, para la aplicación de la categoría de causalidad a objetos determinados y no meramente a un objeto en general no es suficiente la sola determinación a priori de la forma sensible de tales objetos, sino que tal aplicación depende esencialmente de la materia dada a posteriori. Por ello, Maimon indica:

Ahora bien, podría creerse que también la posibilidad de proposiciones sintéticas $a$ priori puede ser entendida, pues si yo juzgo, en un caso particular, que $a$ es causa de $b$, entonces esta proposición particular tiene su fundamento en una universal, a saber: lo que acontece (si es que ha de tener realidad objetiva) debe tener una causa; consecuentemente puedo asimismo aquí saber de antemano que también $b$ tendrá una causa. Pero debe repararse en que en el juicio particular se encuentran determinaciones que no están contenidas en el universal: que a $a$ no debe simplemente seguirle algo que esté con él

$14 \mathrm{VT}, 40$.

15 VT, 201. 
en relación de efecto en general, sino que este efecto puede sólo ser $b$ y no otro; pero esto no puedo determinarlo a priori. ${ }^{16}$

A primera vista, ésta no parece ser una verdadera objeción a la doctrina kantiana. En efecto, el propio Kant sostiene que el principio de causalidad no es condición suficiente de las leyes causales particulares. Kant afirma más bien que tal principio es una condición necesaria de las mismas. ${ }^{17}$ Sin embargo, el punto que señala Maimon es que no siendo el principio de causalidad el fundamento de aquello que hace a una cierta ley causal una ley causal particular, lo particular en ella permanece, para nosotros, sin fundamento alguno:

Yo ya he indicado antes que la proposición universal: "todo tiene su fundamento o su causa" no suministra ningún fundamento de la proposición particular: " $a$ es el fundamento o la causa de $b$." En consecuencia esta proposición particular no tiene (al menos en lo que respecta a nuestra conciencia) fundamento alguno. Las proposiciones analíticas particulares (p.e., un triángulo es igual a sí mismo) adquieren su carácter de ser correctas simplemente mediante la proposición universal (toda cosa es igual a sí misma). Por el contrario, en el caso de las proposiciones sintéticas es al revés: la proposición universal adquiere su carácter de ser correcta meramente porque sin ella no podríamos tener proposiciones particulares de este tipo (que sin embargo tenemos); por lo tanto el universal contiene meramente el fundamento de lo universal en la proposición particular; pero lo particular en ella no tiene ningún fundamento en absoluto. 18

Para garantizar la aplicabilidad de la categoría de causalidad a un objeto determinado no basta con afirmar, como lo hace el principio de causalidad, que dicho objeto debe tener en general una causa. Tal condición debe desde ya ser satisfecha para que un objeto (o más precisamente, un evento) sea representado como perteneciente a la serie objetiva de la experiencia y no como mero eslabón de la serie subjetiva de las percepciones. Sin embargo, la causa debe contener no sólo el fundamento de lo formal del objeto determinado (su lugar en la serie ordenada del

\footnotetext{
16 VT, 211.

17 "Pero la facultad pura del entendimiento, de prescribir a priori, mediante meras categorías, las leyes a los fenómenos, no se extiende a más leyes que aquellas en las que se basa una naturaleza en general, como conformidad de los fenómenos, en el espacio y en el tiempo, a leyes. Las leyes particulares, por concernir a fenómenos empíricamente determinados, no pueden deducirse enteramente de ellas, aunque están, todas, sujetas a aquéllas. Debe concurrir la experiencia para conocer, en general, estas últimas; pero solamente aquellas leyes a priori dan enseñanza acerca de la experiencia en general y de aquello que puede ser conocido como objeto de ella." B 165. Todas las citas de la $\mathrm{KrV}$ han sido tomadas de: Kant (2009).

18 VT, 212 - 213. Como dijimos, según Maimon, la aplicación de la causalidad y en general de las categorías a objetos particulares no es explicada por la doctrina de Kant, sino más bien presupuesta. Presuponemos la proposición particular y aceptamos la universal porque ésta es condición necesaria de aquélla.
} 
tiempo) sino también el fundamento de lo material de tal objeto (su ser así y no de otro modo). En este sentido, Maimon subraya que "causa es algo, mediante lo cual otro algo es determinado; pero determinado no significa meramente puesto, sino puesto de modo determinado."19 La doctrina de Kant, a través de su teoría del esquematismo, demuestra la necesidad de la estructuración categorial del tiempo puro y, en particular, establece que el orden de la sucesión temporal resulta de la aplicación de la categoría de causalidad a la multiplicidad temporal pura. ${ }^{20}$ Con ello se establece que es necesario que la causa de un evento suceda antes que el evento en cuestión. El punto es que esta condición necesaria para que algo sea causa (es decir, para que la categoría se aplique a un objeto determinado) no es suficiente. El efecto debe ser no sólo puesto después de la causa, sino puesto después de modo determinado. Pero la mera estructuración categorial del tiempo puro deja sin explicación cómo un elemento anterior en el orden del tiempo puede poner de modo determinado a otro elemento posterior, pues mediante el sólo ordenamiento del tiempo puro no se comprende cómo la causa puede causar el ser así y no de otro modo del efecto. ${ }^{21}$ De tal manera, "en los objetos de la experiencia podemos conocer meramente la relación de causa y efecto, pero no los miembros de esta relación (qué es lo que sea causa y qué efecto)."22 La doctrina de Kant explica, en el mejor de los casos, la subsunción categorial de un objeto en general, provisto de meras determinaciones formales (entre las que se cuenta su lugar en la serie ordenada del tiempo), pero no la aplicación de las categorías a objetos determinados, porque la estructuración categorial del tiempo puro deja sin explicación la posibilidad de la conexión de los fenómenos según su materia. ${ }^{23}$

\section{La respuesta de Maimon a la pregunta quid juris}

Según la doctrina de Kant, la materia del fenómeno es aquello que sólo nos puede ser dado, es decir lo que nosotros pasivamente recibimos. Pero, precisamente por ello, la materia resulta totalmente heterogénea respecto de la espontaneidad del sujeto. El entendimiento debe aplicar sus funciones de enlace a una multiplicidad sensible, que él no produce y que por ello, según Maimon, incluso si aceptamos la doctrina del esquematismo, no puede enlazar más que según su forma. Como

\footnotetext{
19 VT, 56.

20 B184 - B185.

21 No se exige aquí que se establezca a priori una conexión empírica, sino sólo que se establezca a priori la posibilidad de tal conexión, y no sólo según la forma de ésta sino también según su materia. 22 VT, 124.

23 Aquí falta el "tránsito de los conceptos y proposiciones generales y transcendentales, que se refieren a la experiencia en general, a aquellos que se refieren a la experiencia particular". GW II, 519.
} 
hemos visto, la estrategia de Kant es mostrar la necesaria aplicación de las categorías a la forma de la intuición sensible, para luego sostener que la materia de la intuición empírica debe adoptar las determinaciones que la forma sometida a las categorías le impone. La razón es que si la materia no respetara esta estructura categorial de la forma sensible, no me sería dada en absoluto, o al menos no sería nada para mí. Sin embargo, las determinaciones categoriales que la materia así recibe son siempre universales y nunca alcanzan lo particular de lo dado. En el caso de la causalidad, el juicio a priori según el cual todo evento debe tener una causa es insuficiente para determinar cuál es la causa de cierto evento $a$. Cualquier $b$ anterior a $a$ podría en principio serlo. Más bien, es la experiencia aquello sobre lo que se basa la afirmación de que la causa de $a$ es $b$. Por lo tanto, la justificación de la aplicación de la categoría alcanza sólo a su aplicación respecto de $a$ en tanto objeto en general, ya que como tal $a$ tendrá que tener una causa. Pero la aplicación de la causalidad a $a$ como objeto determinado (el juicio " $b$ es la causa de $a$ ") no se justifica, sino que tal aplicación meramente se asume como un hecho, cuya posibilidad no se explica. 24

Para que sea posible justificar la aplicabilidad de las categorías a un objeto determinado y no sólo a un objeto en general, debe entonces explicarse cómo es posible su aplicación a la materia del fenómeno, dado que la demostración kantiana de la necesaria aplicación de las categorías a la forma del mismo no torna esto comprensible. Ahora bien, si la materia del fenómeno tiene su origen en una receptividad ajena a las funciones del pensar, tal explicación es imposible. En efecto, como hemos visto, en esa situación las categorías sólo pueden referirse a lo universal del fenómeno. La aplicabilidad de las categorías a objetos determinados resultaría entonces comprensible sólo si la materia del fenómeno fuera también producto de una espontaneidad. En ese caso, las categorías no enlazarían una multiplicidad ajena al entendimiento sino que participarían en la generación de tal materia por parte de la espontaneidad. Si adoptamos este punto de vista, el carácter dado de la materia no desaparece, pero resulta reinterpretado. Lo dado ya no es lo pasivamente recibido como efecto de una afección, sino aquello cuya ley de generación nos resulta desconocida. 25

La pregunta quid juris no tiene respuesta satisfactoria en los términos en los que Kant la plantea. ${ }^{26}$ Pero, por el contrario, el problema de la aplicabilidad de las categorías tendría solución si aceptáramos que la materia del fenómeno es producto de

\footnotetext{
${ }^{24}$ El juicio " $b$ es la causa de $a$ " sólo contiene la necesidad correspondiente a que $a$ debe tener en general una causa, pero no la conexión necesaria entre $b$ y $a$, porque este enlace se basa en la experiencia y por ello es contingente.

25 Sobre este punto, véase: Herrera (2010).

26 "Según el sistema de Kant, a saber, en el que sensibilidad y entendimiento son dos fuentes completamente distintas de nuestro conocimiento, esta pregunta es, como he mostrado, irresoluble" VT, 40.
} 
una espontaneidad, es decir que es el resultado de una generación conforme a ley y no un hecho último, absoluto e irracional:

Si nuestro entendimiento pudiera producir objetos a partir de sí mismo, según las reglas o condiciones prescriptas por él mismo, sin que se requiriera que algo le fuera dado de algún otro lado, entonces esta pregunta no tendría lugar. ${ }^{27}$

En este punto, se podría objetar que si nuestro entendimiento generara de modo autosuficiente sus propios objetos, él no sería un entendimiento humano, sino uno divino. Sin embargo, Maimon no niega la finitud de nuestro entendimiento. Maimon no sostiene que nuestro entendimiento produce a partir de sí mismo los objetos sensibles, sino sólo afirma el origen de la materia en una espontaneidad como condición de la aplicabilidad de las categorías. El carácter dado de los fenómenos sólo consiste en que nosotros no somos conscientes de la ley de su generación. La espontaneidad en la que se origina la materia del conocimiento es infinita en tanto ella prescribe las reglas de todo lo que nosotros llamamos sensible, o sea en tanto ella es consciente de aquellas reglas que para nosotros son desconocidas. De tal modo, aun cuando el entendimiento humano y el divino sean una misma espontaneidad, el primero se diferencia del segundo porque la espontaneidad es restringida en nuestro caso, pero ilimitada en el de Dios. ${ }^{28}$ Ahora bien, ¿la referencia a un entendimiento infinito no es en general una afirmación trascendente e injustificada? Para responder a esta pregunta, recordemos que tal entendimiento infinito no es más que una idea, o, en otros términos, no es una entidad metafísica sino puramente lógica. Su modo de ser no es el ser sustancia sino el ser condición, en particular, condición de la aplicabilidad de las categorías a objetos determinados. Una vez que dicha condición fue demostrada como tal, la referencia a ella se encuentra plenamente justificada y no implica ninguna afirmación trascendente. Maimon no adscribe realidad objetiva a la idea de un entendimiento infinito considerada en sí, sino que más bien "ella recibe realidad objetiva para nosotros mediante los objetos de la intuición, de diverso modo. Y también al revés: es decir, las intuiciones adquieren realidad objetiva sólo porque ellas en última instancia deben resolverse en esta idea."29

En resumen, según Maimon, para que sea posible justificar la aplicabilidad de las categorías a un objeto determinado y no sólo a un objeto en general, la materia no puede ser meramente recibida sino que debe ser producida por el pensar:

Por lo tanto, el entendimiento no subsume bajo sus reglas a priori algo dado a posteriori, sino que más bien lo deja surgir de acuerdo con estas reglas (la cual, creo, es la

27 VT, 40.

28 VT, 40.

29 VT, 198. 
única forma de responder a la pregunta quid juris de una manera completamente satisfactoria). 30

Lo dado ya no será lo pasivamente recibido como efecto de una afección, sino aquello cuya ley de generación nos resulta desconocida. Lo sensible resultará así un elemento inteligible, pero confusamente representado. Nuestro intelecto se distinguirá sólo en grado de un intelecto infinito capaz de ser consciente de la ley de generación de lo sensible.

Ahora bien, la ley de la generación de lo sensible es el diferencial. ${ }^{31} \mathrm{Si}$ se suspendiese toda síntesis categorial en nuestras representaciones empíricas, según la doctrina de Kant nos encontraríamos con un elemento último no intelectual, el múltiple de la intuición sensible, materia de aquella síntesis cuya forma conceptual habríamos eliminado totalmente. Por el contrario, el límite de una suspensión completa de la acción de nuestro entendimiento no sería para Maimon un elemento heterogéneo respecto de todo concepto, sino más bien la regla intelectual, desconocida para nosotros, según la cual la representación empírica es generada. ${ }^{32}$ Respecto de los diferenciales, Maimon señala:

Ellos empero son tanto en la matemática como en la filosofía meras ideas que no representan objetos, sino el modo de surgimiento de objetos; es decir: ellos son meros conceptos límite, a los que uno se puede acercar, pero nunca alcanzar. Ellos surgen mediante un continuo regreso o disminución de la conciencia de una intuición hasta el infinito. ${ }^{33}$

La relación que la filosofía establece entre lo empírico y la ley de su generación es para Maimon análoga a la que en matemática existe entre una función y su función derivada. La representación empírica es producto de una síntesis tal como una cierta función derivable resulta de la integración de su función derivada. La derivada de una cierta función es una regla que permite determinar en cada punto de la curva que representa su gráfica cuál será el siguiente punto de la misma. Tal curva

\footnotetext{
30 VT, 50.

31 Para un estudio histórico de la noción de diferencial, véase: Boyer (1949), especialmente 187 - 266.

32 Según Maimon, la materia de la intuición no es un "objeto, sino meramente las ideas en las que finalmente la percepción debe ser resuelta." VT, 115.

33 VT, 21 nota. Un diferencial es una idea en tanto ésta "es un método para encontrar el tránsito de la representación o del concepto de una cosa a la cosa misma". VT, 198. Buzaglo parece no reconocer que para Maimon el diferencial es la idea de una regla y no la de un objeto. Buzaglo (2002) $125-128$. Por ello, a pesar de indicar la conexión entre la causalidad y los diferenciales, Buzaglo no puede resolver el problema de la aplicación física del diferencial (Buzaglo (2002) 127) y rechaza asignarles a los diferenciales un lugar central en la doctrina de Maimon (Buzaglo (2002) 125). Por el contrario, veremos que la clave de la fecundidad trascendental de la noción de diferencial reside precisamente en su caracterización como ley.
} 
podrá así ser generada siguiendo la regla provista por la función derivada ${ }^{34}$. Análogamente, el diferencial del objeto sensible es la ley de generación de lo empírico según la cual el fenómeno se produce. Aquello que para nuestro entendimiento es meramente dado debe ser concebido como producido según una ley, del mismo modo que la curva de una función es generada a partir de la función derivada.

Tanto para Kant como para Maimon la tarea del entendimiento es la de enlazar una multiplicidad en una unidad gracias a la subsunción de lo múltiple bajo conceptos. Pero, según Maimon y en contra de la doctrina de Kant, esta tarea sólo es posible si lo múltiple es representado como producto de una espontaneidad, de modo que la unificación buscada sea alcanzada mediante la indicación del modo en el que tal múltiple es producido:

El entendimiento no puede pensar ningún objeto más que como fluyente (con excepción de las formas de los juicios, que no son objetos). Pues, dado que la tarea del entendimiento no es sino pensar, es decir producir unidad en la multiplicidad, él no puede pensar un objeto más que indicando la regla o el modo de su surgimiento, ya que sólo así lo múltiple de éste puede ser llevado bajo la unidad de la regla. Por lo tanto, él no puede pensar ningún objeto como ya producido sino meramente como produciéndose, es decir como fluyente. 35

Mientras que para Kant el concepto enlaza una multiplicidad pasivamente recibida, para Maimon el concepto enlaza una multiplicidad espontáneamente producida, aún cuando nuestro entendimiento no sea plenamente consciente de la regla de tal producción. Esta regla se encuentra en la base de la síntesis intelectual del fenómeno tanto como magnitud extensiva cuanto como magnitud intensiva. ${ }^{36}$ De acuerdo con la doctrina de Kant, todo fenómeno posee una magnitud extensiva, pues como representación espacio-temporal él sólo es posible mediante la representación de sus partes. A la vez, todo fenómeno posee una magnitud intensiva, en tanto lo real (objeto de la sensación) tiene un grado. En ambos casos, el entendimiento sintetiza en una unidad la multiplicidad sensible, ya sea según la categoría de cantidad (en la magnitud extensiva), o según la de cualidad (en la magnitud intensiva). Por su parte, Maimon entiende que la acción del intelecto que produce la unidad de la intuición (tanto en lo extensivo como en lo intensivo) se ejerce, como vimos, sobre un elemento que debe concebirse como originado en una espontaneidad ilimitada,

\footnotetext{
$34 \mathrm{f}(\mathrm{x}+\varepsilon) \cong \mathrm{f}(\mathrm{x})+\varepsilon \mathrm{f}^{\prime}(\mathrm{x})$

35 VT, $23-24$.

36 "Cada representación sensible considerada en sí misma debe ser, como cualidad, abstraída tanto de toda cantidad extensiva como de toda cantidad intensiva. La representación del color rojo, por ejemplo, debe ser pensada sin ninguna extensión finita, pero sin embargo no como un punto matemático sino físico, o como el diferencial de una extensión. Ella debe ser pensada además sin ningún grado finito de la cualidad, pero sí como el diferencial de un grado finito". VT, 21.
} 
pero cuya regla de generación le es desconocida a nuestro entendimiento finito. Esta regla es el diferencial. El diferencial es el límite de la suspensión completa de toda síntesis de agregación y de coalición. ${ }^{37}$ Este límite es inalcanzable para nosotros, pues supone una serie infinita decreciente hasta la desaparición total de la conciencia: "Lo absolutamente primero en la conciencia de una cosa es una mera idea, que nosotros alcanzamos mediante la infinita reducción de la misma, es decir que nosotros nunca alcanzamos en la intuición." $38 \mathrm{El}$ diferencial es una idea que no puede ser exhibida en la intuición y por lo tanto es un noúmeno, es decir un objeto más allá de los límites de la sensibilidad. 39

La doctrina de los diferenciales permite a Maimon explicar la posibilidad de la aplicación de las categorías a objetos empíricos determinados. Frente a lo que sostiene el esquematismo kantiano, las categorías no se aplican a las formas sensibles del espacio y el tiempo para sintetizar la materia dada en esas formas. Más bien, las categorías se aplicarían a otros elementos intelectuales, los diferenciales, para así subsumir indirectamente bajo sí a los objetos empíricos:

Por ejemplo, si yo digo: rojo es diferente de verde, entonces el concepto puro del entendimiento de 'diferencia' no es considerado como relación de cualidades sensibles (pues de otro modo permanece sin respuesta la pregunta kantiana quid juris), sino, o bien según la teoría de Kant como la relación de sus espacios, como formas a priori, o también según la mía, como relación de sus diferenciales, que son ideas a priori de la razón. 40

De acuerdo con la interpretación de Maimon, la teoría de Kant sostiene que la condición de posibilidad de la diferenciación de dos colores es que ambos sean representados en lugares distintos del espacio único. Así, el concepto de diferencia se aplica a las cualidades intuidas indirectamente, mediante su aplicación a la forma de la intuición. Por su parte, Maimon también entiende que el concepto de diferencia no puede aplicarse inmediatamente a las cualidades sensibles: la heterogeneidad entre concepto e intuición lo impide. Pero en oposición a la doctrina kantiana, Maimon sostiene que la aplicación no resulta mediada por determinaciones de la forma de la sensibilidad, sino por los diferenciales, que son representaciones intelectuales. En este caso, la pregunta quid juris no se plantea, porque los conceptos

37 B 201 nota.

38 VT, 190. A su vez, también es inalcanzable la síntesis completa según la regla que indica el diferencial. En efecto, ambas son "conceptos límite de una síntesis, en tanto sin síntesis no es posible conciencia alguna, pero la conciencia de una síntesis completa abarca en sí lo infinito; en consecuencia ella es imposible para una facultad limitada de conocimiento." VT, 190. De tal modo, Maimon sostiene que "en nuestro conocimiento de las cosas comenzamos por la mitad y terminamos nuevamente en la mitad." Ibídem.

39 VT, 23.

$40 \mathrm{VT}, 23$. 
no se aplican a un tipo heterogéneo de representación: la espontaneidad ejerce su acción categorial sobre sus propios productos (los diferenciales). Así, la diferencia de las cualidades sensibles se representa en la intuición mediante la diferencia de sus lugares e instantes, pero su fundamento o razón es la diferencia de las reglas del surgimiento de tales cualidades. La diferencia de lugares e instantes es la representación fenoménica de la diferencia de los diferenciales, que es una diferencia entre noúmenos.

Lo que hace de un objeto empírico un objeto particular es su materia. Según la teoría de Maimon y en contraposición a la de Kant, la particularidad de un objeto no depende de un elemento pasivamente recibido sino de un producto de la espontaneidad: el diferencial. Así, Maimon afirma que "la regla particular del surgimiento de un objeto, o el tipo de su diferencial lo hace un objeto particular y las relaciones entre objetos diversos se originan en las relaciones de sus reglas de surgimiento, o sus diferenciales." 41

Mediante su teoría de los diferenciales, Maimon reformula la doctrina del conocimiento Kant. La sensibilidad proporciona la materia del conocimiento, pero no porque pasivamente la reciba. Lo que llamamos dato sensible es producto de una espontaneidad que actúa, empero, según una regla para nosotros desconocida. Lo dado por la sensibilidad no es como tal objeto de la conciencia. La conciencia surge como resultado de una síntesis llevada a cabo por la imaginación, que produce la unidad de lo múltiple de la intuición. El entendimiento finalmente aplica sus categorías a los diferenciales y establece así las conexiones de los fenómenos, cuya regla de surgimiento tales diferenciales expresan. ${ }^{42}$ Desde la perspectiva de nuestra subjetividad finita, el orden "subjetivo" de las "operaciones de la mente"43 resulta entonces: sensibilidad (sensación), intuición, conceptos del entendimiento y, finalmente, ideas de la razón, con las que se busca la totalidad de los conceptos del entendimiento. Pero, si adoptáramos la perspectiva de un intelecto ilimitado, en el orden anterior desaparecían la sensibilidad y la intuición, porque tal entendimiento sería consciente de la regla de surgimiento de aquello que nosotros llamamos lo dado. El orden considerado desde este otro punto de vista, que ahora Maimon caracteriza como orden "objetivo", sería: ideas del entendimiento (diferenciales), conceptos del entendimiento, ideas de la razón. 44 Estas tres operaciones de la mente son "condiciones de la intuición misma." 45

41 VT, 24.

42 "La sensibilidad por lo tanto provee los diferenciales para una conciencia determinada; la imaginación produce a partir de ellos un objeto finito (determinado) de la intuición; el entendimiento produce a partir de la relación entre estos distintos diferenciales, que son los objetos de él, la relación de los objetos sensibles que surgen de ellos." VT, 23.

43 VT, 49. VT, $203-204$.

44 VT, 50. El orden objetivo es la representación clara y distinta del orden subjetivo. La distinción entre ambos órdenes permite entender por qué Maimon no sólo se refiere al diferencial como funda- 
Mientras que según la doctrina de Kant las categorías se aplican a los objetos empíricos mediante esquemas, según la teoría de Maimon las categorías se aplican a los objetos empíricos cuando el entendimiento piensa los diferenciales de éstos según aquéllas. Así, por ejemplo, al pensar según la categoría de causalidad los diferenciales del calor del fuego y del grado de fluidez de la cera, el entendimiento establece una dependencia funcional entre la temperatura del primero y el estado de agregación de la segunda. ${ }^{46}$ De tal modo, la regla de surgimiento del calor del fuego (es decir, el modo en el que varía su temperatura) es correlacionada con la regla correspondiente a la cera (es decir, el modo en el que la cera varía su estado de agregación). Así, el entendimiento enlaza las representaciones de fuego y cera como causa y efecto, pero no, tal como indicaría la teoría de Kant, meramente según su forma temporal. En este caso sólo se establecería el orden temporal objetivo de la experiencia como calor del fuego-derretimiento de la cera, pero no se comprendería por qué es el calor del fuego (y no, por ejemplo, otro evento simultáneo a éste) la causa del derretimiento de la cera. Más bien, es ahora la materia de las representaciones lo que se sintetiza al ponerse en relación ambos diferenciales. De este modo, el calor del fuego y el derretimiento de la cera son enlazados como causa y efecto porque el surgimiento de cada uno de estos eventos (y no su mero lugar en el tiempo) se pone en la relación de condición y condicionado. Según la teoría de

mento de la intuición, sino también, en ciertos pasajes, como elemento componente de ella. En este último sentido, Maimon afirma que los diferenciales son "unidades determinadas," tal que de su adición sucesiva surge una magnitud finita (VT, 22 nota) Así, las representaciones sensibles se asocian a las siguientes hasta alcanzar el grado necesario para que se vuelvan conscientes, tal como sucede en un movimiento acelerado con las sucesivas velocidades instantáneas (que "no desaparecen sino que siempre se asocian a las siguientes, de lo que surge una velocidad siempre creciente") (VT, 22) Pero esta composición de lo finito a partir de lo infinitamente pequeño es una representación confusa, propia de la perspectiva del problema desde el orden subjetivo de las operaciones de la mente. Por el contrario, desde la perspectiva del orden objetivo, el diferencial no es una parte componente de lo sensible, sino más bien la regla de su generación. Klapp caracteriza los diferenciales de la conciencia como "las partes componentes inconscientes de la conciencia, que sólo en su conjunto constituyen la conciencia y que contienen la regla del surgimiento de la conciencia.” Klapp (1968) 21. Sin embargo, Klapp no explica cómo resolver la evidente tensión entre la concepción del diferencial como, por un lado, parte componente de la intuición y, por el otro, fundamento lógico de ésta.

45 VT, 50. Así, las categorías no son meramente condiciones de posibilidad de la experiencia, como si pudiera haber de todos modos percepción sin ellas. Para Maimon, las categorías son "condiciones de la percepción misma" (VT,120; 144s), pues, en contraposición a la doctrina de Kant -quien sostiene que "la intuición no necesita en modo alguno de las funciones del pensar" (KrV, A91 / B123)-, las intuiciones no podrían ser dadas independientemente de las categorías.

46 "Tal como nosotros producimos en la matemática avanzada, a partir de los diferenciales de distintas magnitudes, las relaciones mismas de estas magnitudes, el entendimiento produce también (por cierto de un modo oscuro), a partir de las relaciones reales de los diferenciales de diversas cualidades, las relaciones reales mismas de estas cualidades. Si por lo tanto se juzga "el fuego derrite la cera," entonces este juicio no se refiere al fuego y a la cera como objetos de la intuición, sino a sus elementos, que son pensados por el entendimiento en la relación de causa y efecto entre sí.” VT, 193. Las categorías son "reglas del modo de surgimiento de los objetos." VT, 152. 
Maimon, la causalidad se aplica directamente a lo particular del objeto empírico determinado y no a lo universal que hace de él un objeto de experiencia en general. Los conceptos del entendimiento "no son, como supone Kant, válidos respecto de objetos de la experiencia como nos aparecen inmediatamente, sino que son válidos meramente respecto de los límites de los objetos de la experiencia (ideas) y mediante éstas son válidos respecto de los objetos mismos de la experiencia." 47 Dado que según esta teoría de Maimon no existe ninguna heterogeneidad entre la materia y la forma categorial, porque ambas son producto de la espontaneidad, la pregunta quid juris no se plantea.

Ahora bien, el análisis desarrollado hasta aquí podría inducirnos a pensar que Maimon brinda una respuesta positiva a la cuestión quid juris, es decir que resuelve el problema que la doctrina de Kant había dejado abierto, estableciendo finalmente una justificación satisfactoria de la aplicación de las categorías a objetos empíricos. Sin embargo, la filosofía transcendental de Maimon determina solamente las condiciones que una aplicación tal debería cumplir, sosteniendo a la vez que tales condiciones no se verifican en nosotros. Es por ello, que Maimon caracteriza su posición como un dogmatismo racional que implica un escepticismo empírico. Consideraremos ahora esta cuestión.

Kant distingue entre la posibilidad de un concepto y la posibilidad de un objeto de la experiencia. 48 La primera consiste en la ausencia de contradicción del concepto en cuestión. Tal posibilidad lógica es una condición necesaria pero no suficiente para la posibilidad de un objeto tal como el que es pensado mediante el concepto. La posibilidad de un objeto de la experiencia exige más que la posibilidad lógica de su concepto, pues no sólo el principio de contradicción debe ser observado, sino que además las condiciones formales de la experiencia (según la intuición y los conceptos) deben ser satisfechas. ${ }^{49}$ Por su parte, según Maimon, la explicación de la posibilidad de un objeto también requiere mostrar que tal concepto puede ser exhibido en la intuición. Sin embargo, aun cuando esta condición resulte satisfecha, la posibilidad del objeto no será todavía alcanzada. Para que dicha posibilidad pueda ser efectivamente afirmada es necesario que se determine además el modo de surgimiento del objeto correspondiente al concepto. ${ }^{50}$ Es el cumplimiento de esta tercera condición, adicional a la ausencia de contradicción y a la satisfacción de las condiciones formales de la experiencia, la que la doctrina de Kant no demuestra. Por el contrario, mediante su teoría de los diferenciales Maimon afirma haber tornado comprensible el modo de surgimiento del objeto sensible correspondiente al concepto, para así afirmar no sólo la posibilidad, sino más precisamente la "necesi-

\footnotetext{
47 VT, 105.

$48 \mathrm{KrV}$, A $220=$ B 268.

$49 \mathrm{KrV}$, A $218=$ B 265 .

$50 \mathrm{VT}, 37$.
} 
dad de la posibilidad" 51 de tal objeto. En otras palabras, Maimon sostiene haber demostrado apodícticamente la posibilidad de que las categorías se apliquen efectivamente a objetos sensibles, mientras que la argumentación de Kant dejaría abierta la cuestión de tal posibilidad. Según Maimon, la pregunta quid juris puede ser satisfactoriamente respondida sólo si aceptamos que "tanto las formas como los objetos mismos de nuestro conocimiento están a priori en nosotros y que esta facultad no consiste meramente en conocer mediante formas pensadas por nosotros objetos que nos son dados, sino en producir mediante estas formas los objetos mismos." 52 Maimon caracteriza este aspecto de su posición como un dogmatismo racional, pues la razón debe producir sus propios objetos de conocimiento, si es que sus conceptos han de poder aplicarse a objetos. Así, Maimon diferencia su sistema del sistema de Kant, que sería también dogmático aunque empírico y no racional, porque en este caso el objeto de conocimiento debe ser provisto a posteriori, es decir por la experiencia. 53

La teoría de los diferenciales establece las condiciones que deben cumplirse para que las categorías puedan aplicarse a objetos empíricos determinados. No obstante, Maimon subraya que aun cuando las cosas puedan encontrarse en las relaciones pensadas mediante las categorías, el que de hecho se encuentren, es una cuestión que permanece abierta. ${ }^{54} \mathrm{La}$ condición que debe ser satisfecha para que las categorías resulten aplicables a objetos empíricos determinados sólo se cumple si el entendimiento es consciente de la regla de generación de lo sensible. Sin embargo esto no es posible más que para un entendimiento infinito. En el caso de un entendimiento finito, como el nuestro, sólo existe la posibilidad de acercarnos paulatinamente a la conciencia de tal regla (porque ella es producto de una espontaneidad que difiere sólo en grado de la nuestra), pero no de alcanzarla. Por lo tanto, el dogmatismo racional nos lleva a un tipo de escepticismo que Maimon denomina escepticismo empírico. En contraposición al escepticismo racional de Kant, que cuestiona la aplicabilidad de las categorías a cosas en sí,55 el escepticismo empírico de Maimon afirma la posibilidad de la aplicación de las categorías a objetos empíricos determinados, pero considera problemática su aplicación efectiva.

Así, mientras que Kant, según Maimon, no logra probar la posibilidad de la aplicación efectiva de las categorías a objetos empíricos determinados, la argumentación del Ensayo establece tal posibilidad y fija dicha aplicación como un punto inalcanzable pero infinitamente aproximable para nuestro entendimiento limitado. ${ }^{56}$

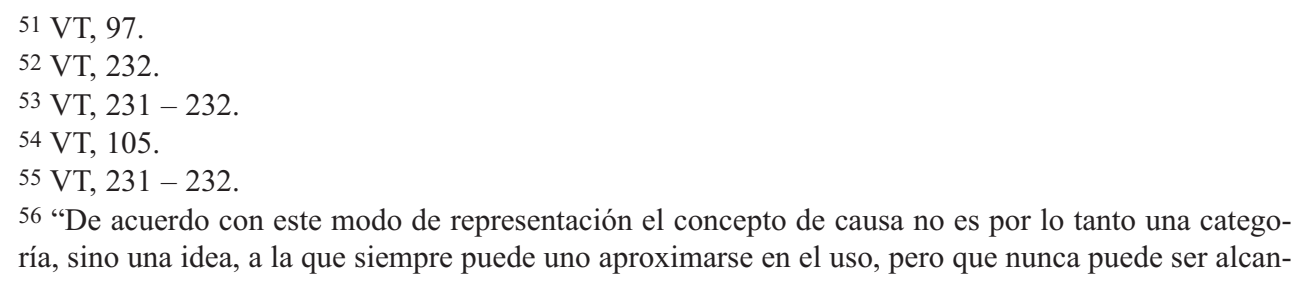
ría, sino una idea, a la que siempre puede uno aproximarse en el uso, pero que nunca puede ser alcan- 


\section{La teoría de los diferenciales y la posibilidad de la metafísica como ciencia}

Hasta aquí hemos reconstruido la interpretación que Maimon realiza del tratamiento kantiano de la cuestión quid juris y hemos presentado su teoría de los diferenciales como respuesta a tal pregunta. Analizaremos para finalizar la relevancia de esta interpretación filosófica del cálculo diferencial respecto de la posibilidad de la metafísica como ciencia.

En la introducción de su Ensayo, Maimon indica que "mediante la reducción de las intuiciones a sus elementos", es decir a sus diferenciales, no sólo se proporciona una clave para responder a la pregunta quid juris sino que, precisamente tal como sucede con la deducción trascendental de Kant, 57 se explica "la posibilidad de una metafísica en general." 58 Éste es el verdadero propósito de la investigación de Maimon. En efecto, Maimon sostiene:

El resultado de esta teoría es por lo tanto éste. Yo afirmo con el señor Kant que los objetos de la metafísica no son objetos de la intuición que puedan ser dados en experiencia alguna. Sin embargo, discrepo de él, en tanto él afirma que ellos no son objetos que puedan ser pensados por el entendimiento como determinados de algún modo. Por el contrario, yo los considero objetos reales que, aunque en sí son meras ideas, sin embargo pueden ser pensados como determinados mediante las intuiciones que a partir de ellos se originan; y mediante la reducción de las intuiciones a sus elementos, estamos en condiciones de determinar nuevas relaciones entre ellos, para así tratar a la metafísica como ciencia. Del mismo modo, nosotros estamos en condiciones de descubrir nuevas relaciones entre las magnitudes mediante la reducción de las magnitudes a sus diferenciales y éstos de nuevo a sus integrales. 59

Según Kant, los objetos de la metafísica se encuentran más allá de los límites de la sensibilidad y con ello fuera de toda experiencia posible. De tal modo, el entendimiento puede pensarlos mediante las categorías, pero tales objetos son así representados sólo mediante conceptos de objetos en general y no resultan conocidos como objetos determinados, más allá de la caracterización puramente negativa, llevada a cabo mediante juicios infinitos, de que ninguna propiedad sensible puede corresponderles. 60 Por el contrario, la doctrina de Maimon de los diferenciales no sólo permite explicar la posibilidad de que las categorías se apliquen a objetos

zada." GW III, 192. En definitiva, la realidad objetiva de las categorías adquiere el carácter de una ficción: aun cuando nuestro entendimiento no pueda concebir la aplicación de las categorías como un hecho, todo ocurre como si lo fuera. VT, 233.

57 Según Kant, "aquella deducción debe decidir primero sobre la posibilidad de una metafísica." Prol AA 4: 260.

58 VT, 11.

59 VT, 110.

60 Véase: Caimi (1998). 
empíricos determinados, sino que permite a la vez determinar relaciones entre noúmenos a partir del conocimiento de los fenómenos que ellos fundamentan. En otras palabras, podría decirse que Maimon muestra al diferencial como ratio essendi de la intuición, pero a la vez a la intuición como ratio cognoscendi del diferencial. Los objetos de la metafísica son ideas, pero Maimon no las considera objetos incognoscibles, como lo hace Kant. Maimon sostiene que se trata de objetos no de la intuición, pero sí del entendimiento "que pueden ser conocidos por nosotros como objetos determinados del pensar, mediante sus esquemas (lo que de ellos es dado en la intuición), aun cuando no en sí (directamente)."61 Una posibilidad análoga de establecer un tránsito desde el conocimiento de lo sensible al de lo suprasensible se verifica en la ciencia físico-matemática: 62 por ejemplo, la velocidad instantánea de un móvil (en tanto magnitud intensiva y función derivada de la trayectoria) puede determinarse a partir de la trayectoria del móvil (en tanto magnitud extensiva y función integral de la velocidad), considerando un espacio y un tiempo infinitamente pequeños. ${ }^{63}$ La magnitud de la velocidad instantánea es "una cantidad determinada que no puede conocerse mediante la velocidad en sí, sino meramente mediante su efecto, es decir mediante el espacio que un cuerpo recorrería con esta velocidad (si ella no se modificara)." 64 Sin embargo, "ni la duración del movimiento, ni el espacio recorrido en ese tiempo pertenecen a la esencia de la velocidad". La velocidad instantánea de un móvil no es directamente un objeto de la intuición, es decir no es un fenómeno, pero puede ser conocida mediante aquello en la intuición que tiene su fundamento en tal velocidad: la distancia recorrida en un tiempo determinado. Sin embargo, para ello debe eliminarse toda síntesis de la imaginación hasta alcanzar el límite de lo inextenso: se debe "hacer abstracción" de toda cantidad finita de espacio y tiempo. En otras palabras, la velocidad instantánea se obtiene al tomarse el límite de la velocidad media cuando el intervalo temporal considerado tiende a cero. Pero este proceso de "reducción" del espacio recorrido y de la duración del movimiento a un "espacio infinitamente pequeño y a un tiempo infinitamente pequeño" 65 no es sino la diferenciación de la función trayectoria. 66 De tal modo, la trayectoria es a la velocidad instantánea como la integral al diferencial y como el fenómeno al noúmeno. El teorema fundamental del cálculo, que establece la integración como la acción inversa de la diferenciación (vinculando los problemas en principio independientes del cálculo de tangentes y el cálculo de áreas) expresa así en términos matemáticos la relación metafísica entre lo fenoménico y lo nouméni-

\footnotetext{
61 VT, 198.

62 Recordemos que nuestra facultad de conocimiento siente un "impulso irrefrenable" de extender los límites del mundo sensible y encontrar "un tránsito del mundo sensible al inteligible.”VT, 184.

$63 \mathrm{VT}, 159$.

$64 \mathrm{VT}, 159$.

$65 \mathrm{VT}, 159$.

66 A su vez, la trayectoria resulta la integral de la velocidad.
} 
co. El tránsito de lo sensible a lo suprasensible, del fenómeno al noúmeno, es análogo a la operación matemática de diferenciación. El tránsito inverso, de lo suprasensible a lo sensible, del noúmeno al fenómeno, es análogo a la integración.

Para Maimon, la metafísica es un conocimiento nouménico, pero no enfrentado al conocimiento fenoménico, porque es más bien el conocimiento del modo en el que el noúmeno funda la realidad fenoménica. La metafísica no es una ciencia de cosas en sí, en tanto substratos transcendentes y heterogéneos respecto de todo fenómeno en nosotros, ${ }^{67}$ sino más bien una ciencia de cosas en sí como "ciencia de los límites de los fenómenos (ideas)" 68 . Así entendida, la "metafísica no sólo es posible, sino que es incluso necesaria, porque de otro modo ningún conocimiento de un objeto sería posible en general". ${ }^{69}$ Tal metafísica fundante de todo conocimiento objetivo se distingue sin embargo de la filosofía transcendental de Kant en que para esta última el conocimiento del fundamento del fenómeno alcanza sólo su forma, pero no su materia. En otras palabras, para Kant el conocimiento a priori de objetos es conocimiento a priori de la forma gracias a la cual los objetos de la experiencia son objetos en general. El fundamento determinable de la forma de los objetos empíricos es el sujeto trascendental. El fundamento indeterminable de la materia de esos objetos es la cosa en sí. Por el contrario, Maimon propone una ciencia del noúmeno, como fundamento tanto de la materia como de la forma del fenómeno. La reducción de las intuiciones a sus elementos diferenciales posibilitaría esta metafísica como ciencia. 70

\section{Bibliografía}

Atlas, S., From Critical to Speculative Idealism. The Philosophy of Salomon Maimon (La Haya 1964).

Bergmann, S.H., The Philosophy of Solomon Maimon. Jerusalem, 1967.

Boyer, C., The History of the Calculus and its Conceptual Development. New York, 1949.

Buzaglo, M., Solomon Maimon: Monism, Skepticism, and mathematics. Pittsburgh, 2002.

CAIMI, M., "A função dos juízos infinitos". Studia Kantiana, 1, 1998, 151-161.

CAimi, M., "The schema of quantity" en Anita Leirfall; Thor Sandmel (coords.), Enhet $i$ mangfold. Festskrift til Johan Arnt Myrstad i anledning 60arsdagen. Oslo, 2009, 77-95.

\footnotetext{
67 Maimon (1790) 250 - 251.

68 Ibídem, 239. Véase también: GW III, 201.

69 Maimon (1790) 239.

70 El presente trabajo contiene resultados del proyecto FONDECYT 1140112.
} 
CAimi, M., "Der Gegenstand, der nach der Lehre vom Schematismus unter die Kategorien zu subsumieren ist" en Stefano Bacin et al. (eds.), Akten des XI. Kant-Kongresses. Berlin, 2013, 147-162.

CASSIRER, E., Das Erkenntnisproblem in der Philosophie und Wissenschaft der neueren Zeit III, en Gesammelte Werke. Hamburg, 2000, vol. 4.

DufFY, S., "Maimon's Theory of Differentials as the Elements of Intuitions", International Journal of Philosophical Studies, 22 (2), 2014, 228 - 247.

EHRENSPERGER, F., Einleitung en Maimon, 2004.

EngstLer, A., Untersuchungen zum Idealismus Salomon Maimons. Stuttgart, 1990. GuÉroult, M., La philosophie transcendentale de Salomon Maimon. Paris, 1929.

Herrera, H., "Salomon Maimon's Commentary on the Subject of the Given in Immanuel Kant's Critique of Pure Reason". The Review of Metaphysics, 63, 2010, 593-613.

Hoyos, L.E., El escepticismo y la filosofía trascendental. Santafé de Bogotá, 2001. KANT, I., Crítica de la razón pura. Estudio preliminar, traducción y notas de Mario Caimi. Buenos Aires, 2009.

Kauferstein, Ch., Transzendentalphilosophie der Mathematik. Stuttgart, 2006. Eckhard Klapp, Die Kausalität bei Salomon Maimon. Maisenheim am Glan, 1968.

Kroner, R., Von Kant bis Hegel, Bd. 1. Tübingen, 1921.

Kuntze, F., Die Philosophie Salomon Maimons. Heidelberg, 1912.

Matherne, S., "Kant and the Art of Schematism". Kantian Review, 19 (2), 2014, 181-205.

Maimon, S., Versuch über die Transzendentalphilosophie. Hamburg, 2004.

Maimon, S., Gesammelte Werke [GW], Valerio Verra (ed.). Hildesheim, 2003.

MAimon, S., "Antwort des Hrn. Maimon auf voriges Schreiben", Berlinischen Journal fûr Aufklârung, IX/1, (1790). Reproducido en: Maimon, 2004, 239251.

Thielke, P., "Intuition and Diversity: Kant and Maimon on Space and Time" en Gideon Freudenthal (ed.), Salomon Maimon: Rational Dogmatist, Empirical Skeptic. Dordrecht, 2003, 89-124.

ZAC, S., Salomon Maïmon. Critique de Kant. Paris, 1988.

Hernán Pringe

CONICET-UBA (Argentina) / Universidad Diego Portales, Santiago (Chile) hpringe@gmail.com 